\title{
Analysis of optimal harvesting of a prey-predator fishery model with the limited sources of prey and presence of toxicity
}

\author{
Sutimin $^{1, *}$, Siti Khabibah ${ }^{1}$, and Dita Anies Munawwaroh ${ }^{1}$ \\ ${ }^{1}$ Department of Mathematics, Faculty of Sciences and Mathematics, Diponegoro University, Semarang - Indonesia
}

\begin{abstract}
A model of prey and predator species is discussed to study the effects of the limited prey density and presence of toxicity. The model is studied for sustainable optimal harvesting. The existence of equilibrium points is analyzed to find the stability of coexistence equilibrium, and use Pontryagin's maximal method to obtain the sustainable optimal harvesting. The results show that the optimal harvesting is obtained from the solution of optimal equilibrium. The toxicity factor decreases the sustainable harvesting.
\end{abstract}

Keywords: Prey-predator; harvesting; stability; equilibrium

\section{Introduction}

In freshwater ecosystems especially in Indonesia, occurred the threat of extinction for diversity and commercial fish that are caused by introducing red devil fish (Amphilophus Labiatus) into the ecosystem. Red devil fish introduced into this ecosystems through human activities for fish cultivation. The population of red devil fish become abundantly and uncontrolled. In originally, red devil species come from Nicaraguan lakes. Now, red devil fish proliferate fast and become predator for original fish that have higher commercial value $[1,2]$. Although, red devil fish has the commercial values but it has no more profit than other original fish, such as tilapia, goldfish. The impact of red devil invasion in freshwater ecosystem is the decreasing of original fish production.

Recently, a number of studies for harvesting fisheries model have been explored. A competition model of two species was studied by Clark et al [2], to analyse the optimal problem in harvesting. The problem also studied to determine the policy of harvesting [3-7].

Next studies, Li et al. [8] studied a model preypredator with logistical growth in prey population without incorporating the harvesting effect in their model. Other model was proposed by Das et al. [9] with the predator growth was in the form of logistical model and the functional response was bilinear form. Das et al. [10] studied other prey-predator model with the prey and predator growths were the logistical model. They considered the limited sources of prey and modelled as functional response of predator to analyse the optimal results in harvesting. Sutimin et al. [11] studied a fishery model of 2-prey and 1-predator for harvesting of these species. They used a logistic model for the growth of each species.

Here, we modify a model of prey predator species with combined harvesting of each species. We incorporate the effect of toxicity in the ecosystem leading to decreasing of population. The growth rate of the population is used logistic type, and the function of response depend on the limited prey population. The global stability of coexistence states and the optimal problem are analysed.

\section{Mathematical model}

In an ecosystem, we assume that there is a toxicity in the freshwater caused by such as damage of ecosystem, industrial waste leading to decreasing of population. The model of prey-predator is developed from logistic growth model and limited of prey population. The model is given as follows,

$$
\begin{aligned}
& \frac{d x}{d t}=r_{1} x\left(1-\frac{x}{K_{1}}\right)-\frac{\alpha_{12} x y}{A+x}-\beta_{1} x-q_{1} E x \\
& \frac{d y}{d t}=r_{2} y\left(1-\frac{y}{K_{2}}\right)+\frac{\alpha_{21} x y}{A+x}-\beta_{2} y-q_{2} E y
\end{aligned}
$$

where $x(t), \mathrm{y}(t)$ indicate the population of prey and predator species, and it increase at rates $r_{1}$ and $r_{2}$, respectively. The carrying capacity of prey and predator,

\footnotetext{
$\overline{* \text { Corresponding author: sutimin } @ \text { undip.ac.id }}$
} 
denoted $K_{1}$ and $K_{2}$. Due to the limitation of prey density and toxicity in freshwater ecosystem, the growth of prey and predator populations is inhibited. Due to the consumption for predator, prey population diminishes at the constant rate $\alpha_{12}$ inhibited with half-saturation constant of prey density $A$. Due to toxicity, prey and predator populations die at the constant rates $\beta_{1}, \beta_{2}$, respectively. Predator population increases at a constant rate $\alpha_{21}$ due to the support of food source of prey and $q_{i}, i=1,2$ are the catching level of prey and predator biomasses, respectively, with the effort unit for biomass harvesting, denoted $E$.

\section{Model Analysis}

The existence and stability of coexistence and bionomic equilibrium solution for the system are analysed in the next sub section.

\subsection{Existence of steady state}

There are some possibility of equilibrium states that it is given as follows.

$P_{0}(0,0)$, the state of extinction of two species.

$P_{1}\left(0, y_{1}^{*}\right)$, the state for survival predator population with prey species extinction, where

$y_{1}^{*}=\frac{K_{2}\left(r_{2}-q_{2} E-\beta_{2}\right)}{r_{2}}$, for $r_{2}>q_{2} E+\beta_{1}$.

$P_{2}\left(x_{1}^{*}, 0\right)$, the state for survival prey species and predator species extinction, where

$x_{1}^{*}=\frac{K_{1}\left(r_{1}-q_{1} E-\beta_{1}\right)}{r_{1}}$, for $r_{1}>q_{1} E+\beta_{1}$.

$P_{3}\left(x^{*}, y^{*}\right)$, the state for both prey and predator exist,

where $y^{*}=\frac{\left(K_{1} r_{1}-E K_{1} q_{1}-x^{*} r_{1}-K_{1} \beta_{1}\right)\left(A+x^{*}\right)}{K_{1} \alpha_{12}}$

and $x^{*}$ is the solution of polynomial

$a_{3} x^{3}+a_{2} x^{2}+a_{1} x+a_{0}=0$

where,

$$
\begin{aligned}
a_{3}= & r_{1} r_{2}, \\
a_{2}= & r_{2}\left(E K_{1} q_{1}+2 A r_{1}+K_{1} \beta_{1}-K_{1} r_{1}\right), \\
a_{1}= & A^{2} r_{1} r_{2}+2 K_{12} r_{2}\left(q_{1} E+\beta_{1}-r_{1}\right) A- \\
& K_{1} \alpha_{12}\left(q_{2} E-\alpha_{21}+\beta_{2}-r_{2}\right) K_{2} \\
a_{0}= & -A K_{1} \alpha_{12}\left(q_{2} E+\beta_{2}-r_{2}\right) K_{2}+
\end{aligned}
$$

$$
A^{2} K_{1} r_{2}\left(q_{1} E+\beta_{1}-r_{1}\right) .
$$

The polynomial has exactly one positive root of $x^{*}$, when

$$
1<\frac{A r_{2}\left(r_{1}-q_{1} E-\beta_{1}\right)}{\alpha_{12} K_{2}\left(r_{2}-q_{2} E-\beta_{2}\right)}, K_{1} r_{1}<K_{1}\left(q_{1} E+\beta_{1}\right)+2 A r_{1} .
$$

\subsection{The stability analysis of equilibria}

Here, we analyse coexistence equilibrium for global stability given as follows.

Theorem 3.1: The stability of coexistence point $P_{3}\left(x^{*}, y^{*}\right)$ is globally asymptotically stable (GAS) if it is hold, $r_{1}>K_{1} \alpha_{12} y^{*}$.

Proof: We define a Lyapunov function

$V(x, y)=x-x^{*}-x^{*} \ln \left(\frac{x}{x^{*}}\right)+h\left(y-y^{*}-y^{*} \ln \left(\frac{y}{y^{*}}\right)\right)$

Differentiating $V$ due to $t$ is ,

$\frac{d V}{d t}=\frac{d x}{d t}-\frac{x^{*}}{x} \frac{d x}{d t}+h\left(\frac{d y}{d t}-\frac{y^{*}}{y} \frac{d y}{d t}\right)$

Consider that $r_{1}\left(1-\frac{x^{*}}{K_{1}}\right)-\frac{\alpha_{12} y^{*}}{A+x^{*}}-q_{1} E-\beta_{1}=0$ and

$r_{2}\left(1-\frac{y^{*}}{K_{2}}\right)-\frac{\alpha_{21} x^{*}}{A+x^{*}}-q_{2} E-\beta_{2}=0$, then

$$
\begin{aligned}
\frac{d V}{d t} & =\left(x-x^{*}\right)\left(r_{1}\left(1-\frac{x}{K_{1}}\right)-\frac{\alpha_{12} y}{A+x}-r_{1}\left(1-\frac{x^{*}}{K_{1}}\right)+\frac{\alpha_{12} y^{*}}{A+x^{*}}\right) \\
& +h\left(y-y^{*}\right)\left(r_{2}\left(1-\frac{y^{*}}{K_{2}}\right)+\frac{\alpha_{21} x^{*}}{A+x^{*}}-r_{2}\left(1-\frac{y^{*}}{K_{2}}\right)-\frac{\alpha_{21} x^{*}}{A+x^{*}}\right) .
\end{aligned}
$$

We have,

$$
\begin{aligned}
\frac{d V}{d t}= & -\frac{\left(r_{1}-\alpha_{12} y^{*} K_{1}\right)}{K_{1}}\left(x-x^{*}\right)^{2}-\frac{h}{K_{2}}\left(y-y^{*}\right)^{2}+ \\
& \left(\left(h \alpha_{21}-\alpha_{12}\right) A-\alpha_{12} x^{*}\right)\left(y-y^{*}\right)\left(x-x^{*}\right) .
\end{aligned}
$$

By choosing $h=\frac{\alpha_{12}\left(A+x^{*}\right)}{\alpha_{21} A}$, it is concluded that

$$
\frac{d V}{d t}<0 \text {. The proof is completed }
$$

\subsection{Bionomic Equilibrium point}

The term is meant as the equality of total revenue and maximum cost of the harvesting efforts.

$$
\begin{aligned}
\text { For } & \frac{d x}{d t}=0 \text {, we have } \\
& r_{1}\left(1-\frac{x}{K_{1}}\right)-\frac{\alpha_{12} y}{A+x}-q_{1} E-\beta_{1}=0
\end{aligned}
$$

and $\frac{d y}{d t}=0$, we have 


$$
r_{2}\left(1-\frac{y}{K_{2}}\right)+\frac{\alpha_{21} x}{A+x}-q_{2} E-\beta_{2}=0
$$

Eliminating $E$ from (3) and substituting into (4), we obtain

$$
\begin{aligned}
r_{2}\left(1-\frac{y}{K_{2}}\right)+\frac{\alpha_{21} x}{A+x}-\beta_{2}-\frac{q_{2}}{q_{1}} r_{1} & \left(1-\frac{x}{K_{1}}\right) \\
+ & \frac{q_{2}}{q_{1}}\left(\frac{\alpha_{12} y}{A+x}+\beta_{1}\right)=0
\end{aligned}
$$

The above equation is called the biological equilibrium. The equilibrium bionomic $\left(x_{\infty}, y_{\infty}\right)$ is determined by above equation and

$$
p_{1} q_{1} x+p_{2} q_{2} y-C=0
$$

it fulfils the condition $\pi=T R-T C$ equals to zero, where $T R$ : total revenue from selling of biomass, $T C$ : total cost for harvesting. Substituting $y$ in above equation into equation (5), we can obtain the solution of $x$ that fulfils the equation

$$
\begin{aligned}
& A_{2} x^{2}+A_{1} x+A_{0}=0 \\
A_{2}= & \frac{r_{1}}{K_{1} q_{1}^{2} p_{1}}+\frac{r_{2}}{p_{2} q_{2}^{2} K_{2}}, \\
A_{1}= & \frac{A r_{1}+K_{1}\left(\beta_{1}-r_{1}\right)}{K_{1} q_{1}^{2} p_{1}}-\frac{\alpha_{12}}{q_{1} p_{2} q_{2}}+\frac{\alpha_{21}}{q_{1} q_{2} p_{1}}, \\
A_{0}= & \frac{A\left(r_{2}-\beta_{2}\right)}{q_{2} q_{1} p_{1}}-\frac{A\left(r_{1}-\beta_{1}\right)}{p_{1} q_{1}^{2}} \\
& +\frac{\alpha_{12} C}{p_{1} p_{2} q_{2} q_{1}^{2}}-\frac{A C r_{2}}{p_{1} p_{2} q_{2}^{2} K_{2} q_{1}} .
\end{aligned}
$$

From the equation (11), we can see that sum of roots is $\frac{-A_{1}}{A_{2}}$ and its product is $\frac{A_{0}}{A_{2}}$. It can be seen that $A_{2}>0$.

The equation (11) has pricely one positive root if $A_{0}<0$

. We have $y_{\infty}=\frac{C-p_{1} q_{1} x_{\infty}}{p_{2} q_{2}}$, so $x_{\infty}<\frac{C}{p_{1} q_{1}}$. The bionomic solution $\left(x_{\infty}, y_{\infty}\right)$, with $x_{\infty}<\frac{C}{p_{1} q_{1}}$ is unique if $A_{0}<0$.

\subsection{Analysis of Optimal Harvesting}

The cost of revenue in time $t$ is defined by,
$\mathfrak{I}=\int_{0}^{\infty} \pi(x, y, E, t) e^{-\delta t} d t$

where $\pi$ is the real income at time $t$ formulated by $\pi(x, y, E, t)=p_{1} q_{1} x E+p_{1} q_{1} x E-C E, \quad \delta$ is the rate of discount annual. To maximize the objective function $\mathfrak{I}$ subject to system (1), we use Pontryagin maximal principle and assume $0 \leq E(t) \leq E_{\max }$. The formulation for Hamiltonian is given as follows,

$$
\begin{aligned}
H=( & \left.p_{1} q_{1} x+p_{2} q_{2} y-C\right) E e^{-\delta t}+ \\
& \lambda_{1}\left(r_{1} x\left(1-\frac{x}{K_{1}}\right)-\frac{\alpha_{12} x y}{A+x}-\beta_{1} x-q_{1} E x\right)+ \\
& \lambda_{2}\left(r_{2} y\left(1-\frac{y}{K_{2}}\right)+\frac{\alpha_{21} x y}{A+x}-\beta_{2} y-q_{2} E y\right)
\end{aligned}
$$

where the variables $\lambda_{1}, \lambda_{2}$ are determined. We have the equations as follows,

$$
\begin{aligned}
& \frac{d \lambda_{1}}{d t}=-\frac{\partial H}{\partial x} \\
& =-p_{1} q_{1} \mathrm{e}^{-\delta t}-\lambda_{2}\left(\frac{\alpha_{21} x y}{(A+x)^{2}}-\frac{\alpha_{21} y}{A+x}\right) \\
& -\lambda_{1}\left(r_{1}\left(1-\frac{x}{K_{1}}\right)+\frac{\alpha_{12} x y}{(A+x)^{2}}-\frac{r_{1}}{K_{1}}-\frac{\alpha_{12} y}{A+x}-\beta_{1}-q_{1} E\right) \\
& \frac{d \lambda_{2}}{d t}=-\frac{d H}{d y} \\
& =p_{2} q_{2} \mathrm{e}^{-\delta t}-\frac{\lambda_{1} \alpha_{12} x}{A+x} \\
& -\lambda_{2}\left(r_{2}\left(1-\frac{y}{K_{2}}\right)-\frac{r_{2} y}{K_{2}}+\frac{\alpha_{21} x}{A+x}-\beta_{2}-q_{2} E\right)
\end{aligned}
$$

To obtain the optimal solution of equilibrium, we take

$$
\begin{aligned}
E & =\frac{1}{q_{1}}\left(r_{1}\left(1-\frac{x}{K_{1}}\right)-\frac{\alpha_{12} y}{A+x}-\beta_{1}\right) \\
& =
\end{aligned}
$$

Eliminating $\lambda_{2}$ in (9) to (10), we obtain the differential equation of $\lambda_{1}$,

$$
\frac{\mathrm{d}^{2} \lambda_{1}(t)}{\mathrm{d} t^{2}}-Q \frac{\mathrm{d} \lambda_{1}(t)}{\mathrm{d} t}+\frac{\alpha_{12} P x}{A+x} \lambda_{1}(t)=M e^{-\delta t}
$$

where,

$P=\frac{\alpha_{21} y}{A+x}-\frac{\alpha_{21} x y}{(A+x)^{2}}, Q=\frac{r_{1} x}{K_{1}}+\frac{r_{2} y}{K_{2}}-\frac{\alpha_{12} x y}{(A+x)^{2}}$, and $M_{1}=P\left(p_{1} q_{1} E \delta+p_{2} q_{2} E\right)$. The quadratic equation in $\eta_{\text {of }}$ (11) is, 


$$
\eta^{2}-Q \eta+\frac{P \alpha_{12} x}{A+x}=0
$$

We can see that the sum of roots is $Q>0$ and its product is $\frac{P \alpha_{12} x}{A+x}>0$. Thus the equation (12) has two positive real roots or complex roots with positive real parts. The solution of (11) is

$\lambda_{1}(t)=C_{1} e^{\eta_{1} t}+C_{2} e^{\eta_{2} t}+\frac{M_{1}}{N} e^{-\delta t}$

where,

$C_{1}, C_{2}$ are constants and $N=\delta^{2}-Q \delta+\frac{P \alpha_{12} x}{A+x} \neq 0$. It is clear that $\lambda_{1}(t)$ is limited for $t \rightarrow \infty$, when $C_{1}=C_{2}=0$.

Thus we have $\lambda_{1}(t)=\frac{M_{1}}{N} e^{-\delta t}$.

In similar way, we obtain $\lambda_{2}(t)=\frac{M_{2}}{N} e^{-\delta t}$, where

$M_{2}=(Q+\delta) q_{2} p_{2}+\frac{x \alpha_{12} p_{1} q_{1}}{A+x}$.

Thus $\lambda_{i}(t) e^{\delta t} \quad i=1,2$ are constant for $t \rightarrow \infty$.

The condition for $H$ maximal is $\frac{\partial H}{\partial E}=0$, so

$\frac{\partial H}{\partial E} \equiv\left(p_{1} q_{1} x+p_{2} q_{2} y-C\right) e^{-\delta t}-\lambda_{1}(t) q_{1} x-\lambda_{2} q_{2} y=0$.

Finally, we obtain,

$\lambda_{1}(t) q_{1} x+\lambda_{2}(t) q_{2} y=e^{-\delta t} \frac{\partial \pi}{\partial E}$.

In (14), it shows the profit value per effort unit in steady state. Inserting the quantity $\lambda_{1}(t), \lambda_{2}(t)$ into (11), we get, $\left(p_{1}-\frac{M_{1}}{N}\right) q_{1} x+\left(p_{2}-\frac{M_{2}}{N}\right) q_{2} y=C$.

The value of equilibrium $E$ is to be obtained from (3) or (4) in term of $M_{1}$, and equation (15) to find the solution of optimal equilibrium $x=x_{\delta}, y=y_{\delta}$. For $\delta \rightarrow \infty$, we have $p_{1} q_{1} x_{\infty}+p_{2} q_{2} y_{\infty}=C$. It leads to $\pi\left(x_{\infty}, y_{\infty}, E\right)=0$. From (15), we have $\pi \equiv\left(p_{1} q_{1} x+p_{2} q_{2} y-C\right) E=\frac{M_{1} q_{1} x+M_{2} q_{2} y}{N}$

We see that $M_{1}, M_{2}$ are the function $O(\delta)$, while $N$ is $o\left(\delta^{2}\right)$, thereby $\pi$ is $o\left(\delta^{-1}\right)$. Therefore $\pi$ decreases when $\delta$ increases, for $\delta \geq 0$. So, we can conclude that for $\delta=0$ the maximum of $\pi$ is obtained.

\section{Numerical results}

The simulation results of the evolution of prey and predator with optimal harvesting is shown in this section. The parameter values for simulation are as follows, $A=300, K_{1}=300, K_{2}=250, r_{1}=2.09, r_{2}=2.07$, $\alpha_{12}=0.03, \quad \alpha_{21}=0.02, q_{1}=0.001, q_{2}=0.01, p_{1}=8$, $p_{2}=5, C=50, \delta=0,05$. From parameter values, it is obtained equilibrium solution of optimal problem satisfying the maximum principle. Calculation results shown that the biological equilibrium point is obtained $P_{3}\left(x^{*}, y^{*}\right)=P_{3}(198,301)$ and the bionomic equilibrium is $R\left(x_{\delta}, y_{\delta}\right)=R(205,280)$ for the optimal harvesting with effort $E=22$ units. The simulations are given as follows.

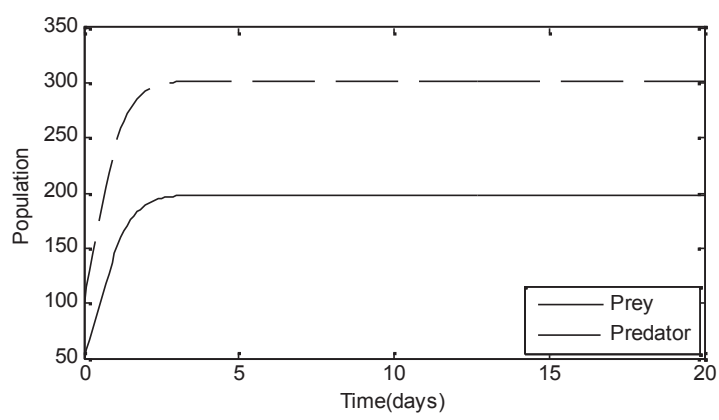

Fig. 1. The evolution of prey and predator populations for biological equilibrium.

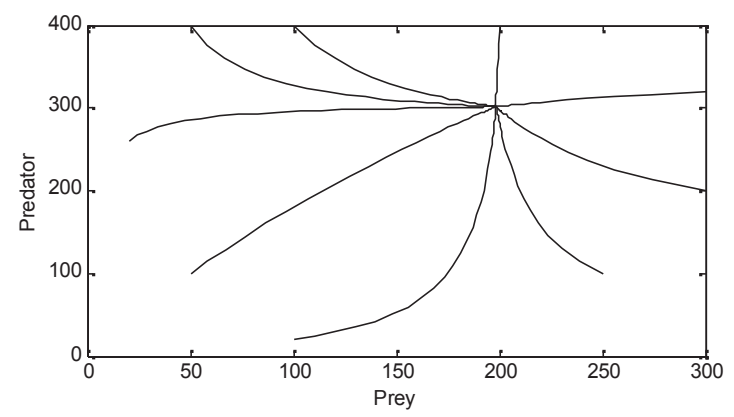

Fig. 2 Prey and predator populations for bionomic equilibrium.

In figure 1, we can see that biological equilibrium closes to the steady state $P_{3}(198,301)$ for harvesting effort $E$ equals to 22 units. In figure 2, it shows that the solutions with the different initial conditions, close to the optimal equilibrium $R(205,280)$. It is shows that for prey population, the optimal equilibrium is larger compered to equilibrium state. On the contrary, for the predator population, the optimal equilibrium is less compered to equilibrium state.

\section{Conclusion}

The behaviour of the system was analysed to study the stability of equilibrium state and the optimal solution including biological equilibrium and bionomic equilibrium solutions. The results shown that coexistence equilibrium is stable. From the simulation results, it was shown that the sustainable harvesting for two species 
(prey and predator) can be obtained. The toxicity factor cause the decreasing of harvesting. So, to prevent coastal ecosystem is needed the efforts for reducing the emission of carbon dioxide in sustainable economic development.

\section{Acknowledgements}

This work is supported by DIPA (2018), Diponegoro University, Semarang, Indonesia.

\section{References}

1. A.K. Sakai, F.W Allendorf, J.S Holt, Lodge D.M, Molofsky J, Kimberly A, S. Baughman, R. J. Cabin, J.E. Cohen, N.C. Ellstrand, D.E. McCauley, P. O'Neil, I.M. Parker, J.N. Thompson, S.G. Weller, Annu. Rev. Ecol. Syst., 32 (2001).

2. M. Clavero, Garcý'a-Berthou E. Trends Ecol Evol 20 (2005).
3. C.W. Clark, John Wiley and Sons, New York,NY, USA, (1990).

4. T. Das, R.N. Mukherjee, K.S. Chaudhuri, App. Math. Modell. 33:228-2292 (2009).

5. T.K. Kar, U.K. Pahari, K.S. Chaudhuri, J. Appl. Math. \& Computing, 19(1-2), 311-326 (2005).

6. R. Zhang, J. Sun, H. Yang, Appl. Math. Sci. 1 : 24812492 (2007).

7. B.R. Reddy, Int. J. Math. Trends and Tech. 4(2), (2013).

8. B. Li, S. Liu, J. Cui, J. Li, Appl. Sci., 151(6): 1-18, (2016)

9. T. Das, R.N. Mukherjee, K.S. Chaudhuri, Appl. Math. Model. 33(5):2282-2292, (2009).

10. T. Das, R.N. Mukherjee, K.S. Chaudhuri, J. Biol. Dyn. 3(5):447-462 (2009).

11. Sutimin, S. Khabibah, D.A. Munawwaroh, E3S Web Conf. 31, 08008 (2018). 